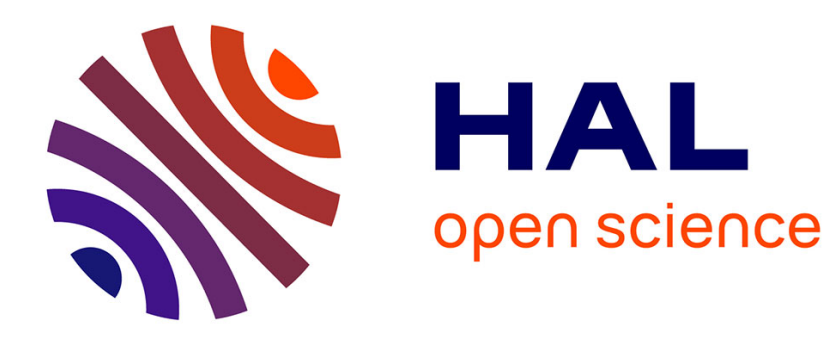

\title{
Inventaire des potins de Manching, de Bavière et de Bohême
}

Bernward Ziegaus

\section{To cite this version:}

Bernward Ziegaus. Inventaire des potins de Manching, de Bavière et de Bohême. Gallia - Archéologie de la France antique, 1995, Les potins gaulois, 52, pp.95-100. 10.3406/galia.1995.3131 . hal-01911271

\author{
HAL Id: hal-01911271 \\ https://hal.science/hal-01911271
}

Submitted on 27 Feb 2020

HAL is a multi-disciplinary open access archive for the deposit and dissemination of scientific research documents, whether they are published or not. The documents may come from teaching and research institutions in France or abroad, or from public or private research centers.
L'archive ouverte pluridisciplinaire HAL, est destinée au dépôt et à la diffusion de documents scientifiques de niveau recherche, publiés ou non, émanant des établissements d'enseignement et de recherche français ou étrangers, des laboratoires publics ou privés.

\section{(이) $\$$}

Distributed under a Creative Commons Attribution - NonCommercial - NoDerivatives| 4.0 


\title{
4.3. Inventaire des potins de Manching, de Bavière et de Bohême
}

\section{Bernward Ziegaus}

\begin{abstract}
Résumé. À Manching, en Bavière du Sud, le nombre des monnaies qui venaient de l'étranger souligne l'importance des contacts avec d'autres régions, même de la périphérie du monde celtique. À Manching et à l'est de cet oppidum, en Bohême jusqu'à la frontière de la Norique, on trouve non seulement des monnaies d'or et d'argent mais aussi des monnaies coulées. C'est un phénomène exceptionnel de trouver des monnaies moins précieuses, qui représentent la monnaie des petites gens, à des centaines de kilomètres de leur lieu de circulation habituel. La plupart des types sont les potins "au sanglier " des Leuci (Scheers pl. 23, 658-671) et les monnaies attribuées aux Sequani. Les potins des Lingones et du type Zürch sont rares, et ceux des Remi, Bellovaci, Treveri, ou Senones ne jouent presque aucun rôle. pour la circulation monétaire. Les analyses métallurgiques de quelques potins trouvés à Manching ont montré que c'est probablement l'élément antimoine (Sb), qui est caractéristique des monnaies coulées de Manching, tandis que dans les potins de provenance gauloise, cet élément n'existe pas, paraît-il. Dans certaines fosses datées de l'horizon La Tène D1 par les fibules et la céramique peinte, on a trouvé quelques pièces des Leuci-Sequani A 1/2 et une monnaie du type Zürch. La fin de l'occupation de l'oppidum de Manching daterait des années 50-30 avant J.-C.
\end{abstract}

Zusammenfassung. Aus der behandelten Region stammen 146 Potinmünzen, zwei Drittel davon allein aus Manching. Die übrigen verteilen sich auf weniger bekannte Siedlungen wie Pollanten, Egglfing oder Stöffling. Es überwiegen die Potinmünzen der Leuker («au sanglier") und der Sequaner. Die Typen der Lingonen sowie der Typ Zürch sind selten, die Potinmünzen der Remer, Bellovacer, Treverer oder Senonen spielen keine Rolle. In Manching gibt es keinen Nachweis für eine Fabrikation am Ont. Verschiedene Typen (Leuker, Sequaner A1/2 und Typ Zürch) sind in Manchinger Cruben mit Fundmaterial der Stufe La Tène D1 vergesellschaftet (Nauheimer Fibel, Feinkammstrichkeramik, bemalte Keramik).

Avec la publication des trésors de Manching et des monnaies celtiques trouvées en Bavière du Sud par H.J. Kellner, il existe maintenant après l'œuvre de $\mathrm{S}$. Scheers qui a étudié les monnaies de la Gallia Belgica, une seconde œuvre qui résume la recherche scientifique et nous donne un catalogue des trouvailles pour une grande région (Kellner, 1990).

Si l'on observe attentivement la répartition des monnaies celtiques dans notre zone, on remarque, que non seulement aux endroits centraux comme par exemple à Manching et à Stradonice, mais aussi dans le reste de la Bavière du Sud et en Bohême circulent des monnaies vcnant dc l'ouest, du sud et de l'est et qui entrent dans la circulation monétaire locale. Tout d'abord la distribution entre les métaux or, argent et potin à Manching est d'un intérêt particulier, parce que ce ne sont pas les précieux statères en or, utilisables pour des paiements d'une affaire commerciale à l'étranger, mais les monnaies en argent qui dominent ainsi que les potins de Gaule et de Suisse, qui constituent $10 \%$ de la circulation totale. Les deux monnaies d'or étrangères (Kellner, 1990, $\mathrm{n}^{\circ} 4,32$ ) par rapport aux sept cent quatre-vingt-dix-neuf monnaies celtiques trouvées jusqu'à 1986 ne jouent aucun rôle dans les trouvailles.
Le nombre de monnaies augmente après la fin de l'inventaire en 1986 dans une proportion incohérente à cause des trouvailles mises au jour à l'aide de détecteurs. Comme les circonstances des trouvailles et les informations ne sont pas contrôlables, je n'ai pas pris en considération ces monnaies dans mes analyses concernant la circulation des potins. J'ai analysé les potins de la publication de Kellner trouvés jusqu'en 1986 (Kellner, 1990) et ceux, qui ont été remis à la Prähistorische Staatssammlung München depuis ce temps-là. Dans mes recherches j'ai essayé de répondre aux questions suivantes.

\section{QUELS SONT LES TYPES DE POTINS TROUVÉS À MANCHING ET DANS LE RESTE DE LA RÉGION EXAMINÉE QUELS SONT LES TYPES LES PLUS FRÉQUENTS?}

On connaît cent quarante et un potins trouvés sur le territoire examiné, soit deux tiers de toutes les pièces venant de l'oppidum de Manching. Ce grand nombre est le résultat d'un examen systématique de l'oppidum pendant des dizaines d'années ce qui donne une bonne vue d'ensemble sur la circulation monétaire d'un site central. Le reste des potins sont des trouvailles anciennes de l'oppi- 
dum de Stradonice (Píc, 1903, pl. 2), des pièces découvertes dans les habitats de Berching-Pollanten en Bavière du Nord (Kellner, 1990, n ${ }^{\circ} 40-943$ ), Egglfing près de Regensburg (inédits : quatre pièces Scheers 658 ff., quatre pièces « ̀̀ la grosse tête » au bandeau lisse type A, une pièce LT 9361), Stöffling, Lkr. Traunstein au bord du lac Chiemsee (deux pièces Scheers 658 sq., une pièce « à la grosse tête " au bandeau lisse type $\mathrm{A}$, trois pièces « à la tête diabolique ") et des trouvailles fortuites de Bavière (Kellner, 1990, n²067, 2078, 2105, 2161 ; Ziegaus, 1989, $\mathrm{n}^{\circ} 19,99,126$ et environ quinze pièces encore inédites du type Scheers 658 ff. et du type «à la grosse tête » au bandeau lisse type A trouvées à Manching depuis 1986) et de Bohême (deux pièces LT 8124, Radomersky, 1955, p. 48, $\mathrm{n}^{\circ} 39$; quatre pièces LT 8124 , Scheers, 1977 , p. $762, \mathrm{n}^{\circ} 143$ et $\mathrm{n}^{\circ} 150$; une pièce IT 9361 et une pièce « à la grosse tête " Píc, 1903, n³6 et 39). Manching se singularise par l'importance numérique des découvertes monétaires et a dû jouer un rôle particulier. La liste des potins différents dans les oppida et les habitats laténiens se répartissent de la manière suivante (tabl. 7).

Cette répartition montre que ce sont essentiellement deux types de potins, qui circulent à Manching, notamment le type Scheers 658-670 et le type «à la grosse tête » au bandeau lisse (Geiser, Gruel, type A). Les potins du type LT 9361 (type Zürch), LT 8319 et 8124 constituent, avec onze pièces, environ $10 \%$ de l'inventaire et jouent un rôle moins important. La circulation de nombreuses monnaies du type Scheers $658 \mathrm{sq}$. et les potins «à la grosse tête " type A n'est pas seulement caractéristique de Manching, mais aussi des autres habitats laténiens en Bavière. Il est plus difficile de faire des rapports concernant la circulation des potins en Bohême, parce que, depuis la parution de la monographie de J.L. Píc sur le Hradiste de Stradonice, presque aucune monnaie n'a été découverte.

Si l'on observe les potins principaux de la région examinée type Scheers 658 sq. et les monnaies du type «à la grosse tête ", on peut constater une concentration de certaines variantes. Une analyse du type Scheers 658 sq. concernant les différents dessins sous le sanglier montre la distribution suivante (tabl. 8).

À l'aide de la diffusion de ces variantes, il est pourtant aujourd'hui encore très difficile de se faire une opinion définitive. La raison est d'une part qu'une grande quantité ne peut pas être identifiée à cause de la corrosion et d'autre part, que les auteurs prennent de moins en moins le soin de dessiner les monnaies.
Tabl. 7. Les différents potins dans la région examinée.

\begin{tabular}{|l|c|c|c|c|}
\hline \multicolumn{1}{|c|}{ Type } & Manching & Bavière & Stradonice & Bohême \\
\hline Scheers 658-570 & 53 & 4 & 3 & 1 \\
\hline Grosse tête A & 40 & 9 & 1 & \\
\hline LT 9361 & 4 & 3 & 1 & \\
\hline LT 8319 & 6 & & & \\
\hline LT 8124 & 1 & & 4 & 2 \\
\hline BN 5687/5674 & 2 & 5 & & \\
\hline LT 7417 & 2 & & & \\
\hline
\end{tabular}

Tabl. 8. Classification d'après Scheers, 1977, p. 712 sq., pl. XXIII, $n^{\circ} 658-670$.

\begin{tabular}{|c|c|c|c|}
\hline Classe & Manching & Stradonice & Autres endroits \\
\hline la & 5 & & 4 \\
\hline Ib & 4 & & \\
\hline Ic & 8 & & \\
\hline Id & 3 & & \\
\hline Ie & 2 & & \\
\hline Ig & 11 & & \\
\hline Ih & & 1 & \\
\hline Ij & 3 & & \\
\hline II & 1 & & \\
\hline Cl. I/II & 15 & & \\
\hline
\end{tabular}

Tabl. 9. Ies potins "à la grosse lêle " d'après la typologie Geiser, Cruel, 1993.

\begin{tabular}{|c|c|c|c|}
\hline Groupe & Manching & Bavière & Bohême \\
\hline A 1 & 17 & 4 & 1 \\
\hline A 2 & 5 & 1 & \\
\hline A $2 / 1$ & 1 & 1 & \\
\hline A 4, 1 & 4 & 1 & \\
\hline A 5 & 2 & & \\
\hline A 6, 1 & 4 & 1 & \\
\hline A 8,1 & 2 & & \\
\hline A var. ? & 5 & & \\
\hline B & & & \\
\hline
\end{tabular}


La conséquence est qu'une répartition sans équivoque dans l'une ou l'autre classe n'est pas possible et pour vingt-trois pièces, donc pour presque la moitié, la classification reste douteuse. L'inventaire de Manching montre que quelques variantes sont rares alors que d'autres sont plus fréquentes. Ce sont par exemple les pièces de la classe Ic avec huit exemplaires et classe Ig avec onze exemplaires, qui dominent. Ces variantes disposent d'un symbole avec trois cintres ou un croc barbelé sous le sanglier.

Les monnaies très fréquentes aussi sont les potins « à la grosse tête ", qu'on a attribués depuis longtemps déjà aux Séquanes. La subdivision de ce type et la reclassification par A. Geiser et K. Gruel (1993) donnent le résultat résumé dans le tableau 9 .

La nouvelle classification prouve que quelques variantes sont plus nombreuses, par exemple le groupe $\mathrm{Al}, 2,4,5,6,1$ et 8,1 , tandis que les monnaies du groupe A6,2 et 6,3, qui disposent d'une roue ou d'un cercle, manquent aussi bien que les pièces des groupes $A 9,10$ et 11 . Les monnaies qu'on doit attribuer au type B au bandeau décoré (B1-B5) manquent aussi.

Il y a quelques particularités pour les pièces du groupe Al et A2 : les monnaies du groupe Al de Manching et de Bavière disposent sans aucun doute des dessins que $\mathrm{A}$. Geiser et $\mathrm{K}$. Gruel ont remarqués (avers : petite tête avec un œil approfondi, un cou peu courbé et un globule dans la bouche; revers : taureau avec une tête baissée et une longue queue, qui se déroule en direction de la tête et dont l'extrémité est tournée vers le haut), mais les pièces analysées ne montrent en aucun cas une légende ou les traces d'une légende derrière la tête de l'avers. Le groupe, que j'ai appelé A2/1 me semble une combinaison des deux variantes. L'avers ressemble au motif du groupe A2 et le revers du groupe $\mathrm{Al}$.

Les monnaies qui appartiennent sans équivoque au groupe A2, qui montrent un taureau avec une tête peu levée et un picd avant formant un angle aigu, existent aussi à Manching, mais elles sont plus rares que les pièces du groupe A. Excepté les exemplaires fréquents du groupe $A$ et les pièces du groupe $B$, qui sont complètement absentes, il reste encore un potin qu'on peut peut-être attribuer au groupe C (cf. Furger-Gunti, von Kaenel, 1976 , p. 54, fig. C; Kellner, 1990, n³0 trouvé à Manching). Nous en connaissons six exemplaires du site de la colline de la Cathédrale à Bâle et deux exemplaires du site de l'Usine à gaz de Bâle (Furger-Gunti, von Kaenel, 1976, p. 56). Une comparaison des dessins montre qu'il y a une certaine ressemblance entre les mon- naies de Bâle et celles de Manching du type $\mathrm{C}$ (cf. aussi Castelin, 1978, $\mathrm{n}^{\circ} 768$ sq.), mais une décision définitive sur l'attribution au type "à la grosse tête " me semble difficile à prendre. Dans le groupe A, il n'y a aucune différence, que le taureau ait la tête baissée ou levée, parce que la quantité des variantes est presque égale et aucune ne dominait dans la circulation monétaire à Manching.

\section{QUELLE EST L'IMPORTANCE DES POTINS DE L'OPPIDUM DE MANCHING, DE BAVIÈRE ET DE BOHÊME POUR LA CIRCU- LATION MONÉTAIRE CELTIQUE?}

Si l'on se rappelle le nombre des monnaies trouvées dans l'oppidum de Manching et la distribution entre les différents métaux, or, argent, bronze et potin et si l'on calcule le pourcentage des potins en considérant toutes les pièces trouvées lors des fouilles, on obtient $10 \%$. Ie plus grand nombre de monnaies trouvées à Manching comprend des monnaies d'argent, les Büschelquinare d'un poids moyen de 1,8 g (Kellner, 1990, tableaux récapitulatifs des types 5 ) et les petites pièces d'argent d'un poids moyen de 0,4 g (Kellner, 1990, tableaux récapitulatifs des types 7 et 8). Il s'agit des types locaux de Manching.

Malheureusement, on ne connaît pas l'évaluation et la relation entre la plus petite monnaie d'argent et le potin, mais on peut estimer, à cause de la quantité, que les monnaies faibles en argent représentent la petite monnaie en circulation à Manching. Si l'on essaie de comparer la valeur de ces monnaies avec celle des potins, on peut soupçonner que les potins possédaient peut-être la même valeur ou une valeur moins importante, et correspondaient donc à un pouvoir d'achat réduit. On peut aussi constater qu'on a accepté les potins comme moyen de paiement sinon on trouverait ces pièces moins fréquemment. Ni les petites monnaies d'argent, ni les potins ne convenaient aux grands mouvements monétaires, bien qu'il y ait aussi des dépôts, qui se composent de petites monnaies d'argent comme par exemple le grand dépôt de Neuses en Haute-Franconie, qui contient non seulement plus de trois cent trente Büschelquinare, mais aussi soixante petites pièces d'argent (Overbeck, in : Kellner, 1990, p. 227-229). Les dépôts de la région examinée sont composés régulièrement d'argent et $d$ 'or et ne contiennent - jusqu'à ce jour - jamais de potins. Par rapport au nombre des monnaies d'argent de Manching, le potin joue un rôle subalterne.

La répartition des potins en dehors de Manching montre (fig. 42), qu'en allant vers l'est, le nombre de 


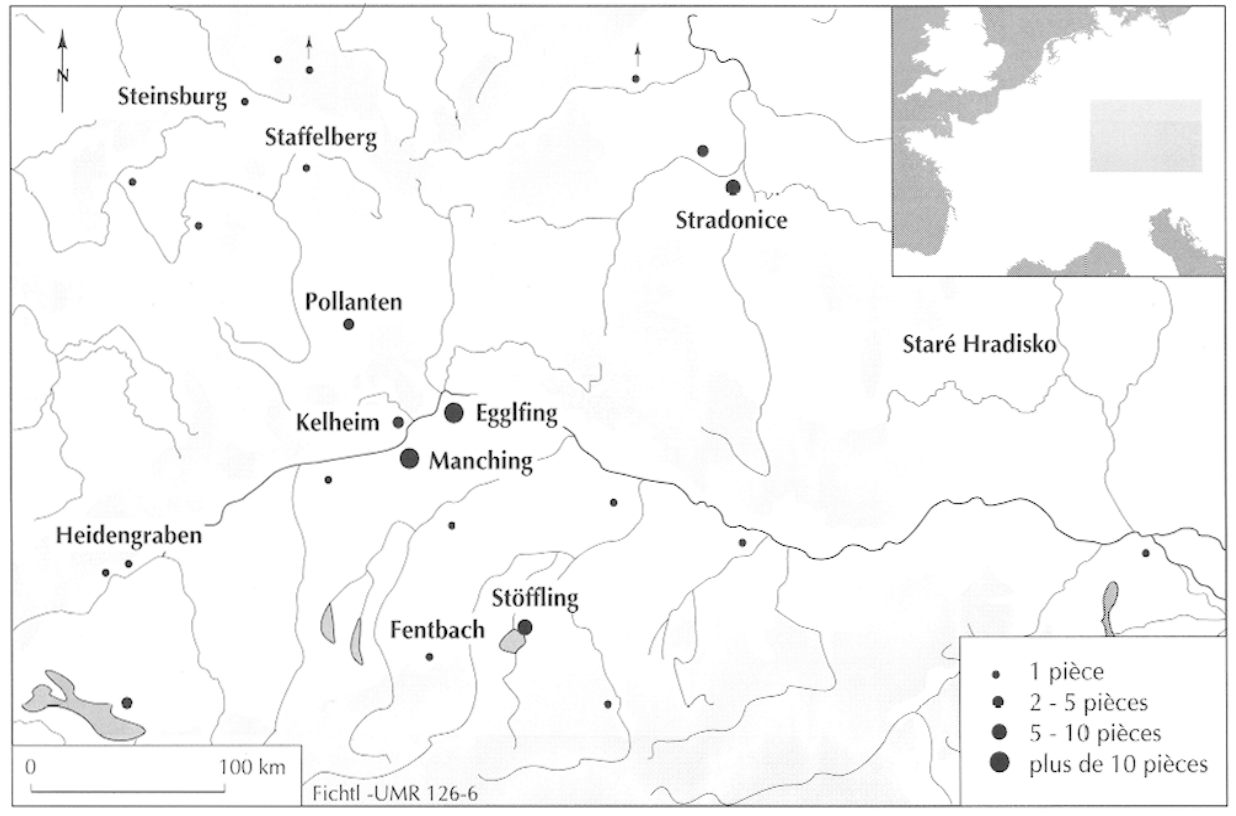

Fig. 42. Répartition des potins en Bavière et en Bohême. potins diminue, mais les trouvailles sont bien documentées. H.J. Kellner a déjà observé ce phénomène dans sa publication (Kellner, 1990, p. 28 sq.) et les nouvelles découvertes semblent confirmer cette tendance. Pourtant il reste étonnant qu'on ait découvert des potins dans l'oppidum de Stradonice et pas seulement une ou deux pièces isolées mais quelques exemplaires des différents types. On y trouve même des types rares à Manching, par exemple une pièce du type I.T 9361 et quatre pièces du type LT 8124 . Sur la carte de répartition, on voit nettement que les potins ont été diffusés très loin en direction de l'est. Il y a une monnaie du type BN 7905 (Scheers 695) à Pirna en Saxe (Koblitz, 1918, n²9), une pièce du type I.T 5629 à Seebergen, Ldkr. Gotha (Neumann, Kellner, 1966, $\mathrm{n}^{\circ} 32$ ), un potin type Scheers 658 et deux du type LT 8124 (Koblitz, 1918, n³4 et Radomersky, 1955, n³9) à Komotov en République tchèque, une monnaie du type "à la tête diabolique * I.T 7414 aux environs de Linz en Autriche (Prokisch, 1993, A. 46) et une monnaie du type LT 7414 à Camuntum (Hahn, 1976, $\mathrm{n}^{\circ} 1$ ).

Toutes ces monnaies prouvent sans aucun doute, qu'une circulation régionale pour certains types est possible, mais qu'il existe aussi des contacts très lointains. Ce phénomène montre que ces monnaies ne prenaient pas le chemin direct de la Gaule et de la Suisse à l'Allemagne centrale, Bohême et Norique, mais passaient plutôt par les grands oppida par exemple Manching, Kelheim ou Stradonice el de là allaient encore vers l'est. Si l'on résu- me les résultats particuliers, il est sûr que la concentration des potins en Bavière se trouve dans la localité de Manching, mais la circulation des monnaies d'argent domine et les potins jouent un rôle moins important. Les monnaies d'argent dominent en Bavière, en Bohême et en Norique.

\section{EXISTE-T-IL DES INDICES D'UNE FABRICATION DE POTINS À MANCHING ET QUEL POINT DE REPÈRE DONNENT LES POTINS TROUVÉS DANS LES FOSSES DE MANCHING?}

Une des questions les plus difficiles est la localisation de la fabrication des potins. Sur l'oppidum de Manching, on a découvert cent quarante moules utilisés pour la production des flans en argent et en or (Kellner, 1990, p. 230-235). U. Zwicker supposait, il y a quelque temps, qu'on utilisait pour la production des potins des moules en plâtre, qui n'existent plus aujourd'hui, parce qu'ils sont détruits à l'inverse des moules en céramique (Zwicker in: Kellner, 1990, p. 252-254).

Manching, seul lieu où on a découvert un grand ensemble de potins, est-il l'endroit où ils ont été coulés? J.-B. Colbert de Beaulieu avait soupçonné que les mêmes types sont produits dans des lieux différents (Colbert de Beaulieu, 1959, p. 25). À cause de la grande masse du type Scheers 658 sq. et du type "à la grosse tête " au bandeau lisse à Manching, on peut penser que la fabrication de ces types était possible sur place. En principe, cette fabrication est possible parce qu'on a seulement besoin de 
modèles d'une qualité utilisable pour faire des répliques. $\mathrm{Au}$ fond, la coulée était réalisable dans chaque atelier de métallurgie.

Si l'on cherche les variantes, ce sont avant tout les monnaies du type Scheers 658 sq. de la classe Ic et Ig, qui sont plus fréquentes que d'autres. Malgré le nombre important des deux classes avec huit et onze exemplaires, on n'a aucune preuve qu'on a fabriqué ces variantes à Manching : il serait plutôt nécessaire d'observer la diffusion. Un indice pour une fabrication serait peut-être la présence de nombreuses pièces de même coulée, ce qu'on peut expliquer par un besoin local de potins à l'oppidum de Manching. Identifier des potins issus des mêmes moules est très difficile et la caractéroscopie, si utile pour les monnaies d'or ou d'argent est difficilement applicable aux potins. En effet, il y a quelques monnaies du type Scheers 658 sq. qu'on peut bien comparer, mais elles ne viennent probablement pas du même modèle (cf. Kellner, $1990, n^{\circ} 8$ et 428 ou $n^{\circ} 439$ et 443 ou $n^{\circ} 7$ et 443 ). Il y a quelques monnaies du type « à la grosse tête " analogues à ce type (Kellner, 1990, n ${ }^{\circ} 458-462$ ou $\mathrm{n}^{\circ} 463$ et 464). En outre, nous connaissons à Manching deux potins usés du type Scheers $658 \mathrm{sq}$. (classe ?) trouvés dans une fosse (Kellner, 1990, $\mathrm{n}^{\circ} 10$ et 12). Des indices concrets d'une coulée comme des moules, des fragments ou des potins défectueux n'existent pas.

Une analyse métallurgique pourrait-elle donner une réponse en faveur d'une fabrication locale des potins à Manching? Un examen de douze pièces type Scheers 658 sq. et de potins "à la grosse tête " (Zwicker, in: Kellner, 1990, p. 255 sq.) montre d'après l'interprétation de U. Zwicker, que la présence ou l'absence de l'élément antimoine donne une indication de la provenance du métal qu'on a utilisé pour la coulée (Zwicker, in : Kellner, 1990, p. 248 sq.). Il est caractéristique de certaines pièces de Manching que l'élément antimoine est nettement présent, alors que les pièces qu'on a trouvćcs en Gaule ne possèdent pas de cet élément. J'ai encore analysé neuf autres potins, qui viennent de l'oppidum de Manching et qui montrent les résultats suivants (tabl. 10).

Ce tableau peut seulement donner une orientation approximative et montre les proportions entre les éléments analysés. J'ai renoncé aux pourcentages exacts en raison du problème des aléas d'une expérience et des erreurs typiques d'analyse des surfaces. Presque toutes les pièces comportent une grande quantité de cuivre (14$53 \%)$, d'étain (15-63\%) et de plomb (11-22\%). Les autres éléments comme le fer, le nickel, l'arsenic et l'an-
Tabl. 10. Analyse par rayons $X$. Composition en surface des potins de Manching (+++ = < $10 \% ;++=2-10 \% ;+=0,1-2 \% ; \mathrm{T}$ $=$ traces $; 0=$ sans détection). $K:$ Invenlaire des monnaies celtiques du Prähistorische Staatssammlung München.

\begin{tabular}{|c|c|c|c|c|c|c|c|}
\hline Type & $\mathrm{Cu}$ & Sn & $\mathrm{Pb}$ & $\mathrm{Fe}$ & $\mathrm{Ni}$ & As & $\mathrm{Sb}$ \\
\hline 1. "grosse tête" A $1 / 2 \mathrm{~K} 576$ & $\bullet \bullet$ & $\bullet$ & $\bullet$ & $\bullet$ & - & $\bullet$ & $\bullet$ \\
\hline 2. "grosse tête " A $1 / 2 \mathrm{~K} 119$ & $\bullet \bullet$ & $\bullet \bullet$ & T & - & - & 0 & 0 \\
\hline 3. "grosse tête " A 1 K 347 & $\bullet \bullet$ & $\bullet \bullet$ & $\bullet$ & $\bullet$ & - & $\bullet$ & $\bullet$ \\
\hline 4. Scheers 660 & $\bullet \bullet \bullet$ & $\bullet \bullet \bullet$ & $\bullet$ & - & - & $\mathrm{T}$ & $\bullet$ \\
\hline 5. Scheers 661 & $\bullet \bullet$ & $\bullet \bullet$ & $\bullet \bullet \bullet$ & $\bullet$ & - & $\bullet$ & $\bullet$ \\
\hline 6. Scheers 664 & $\bullet \bullet$ & $\bullet \bullet$ & $\bullet \bullet \bullet$ & $\bullet$ & - & $\bullet$ & $\bullet$ \\
\hline 7. Scheers 666 & $\bullet \bullet$ & $\bullet \bullet$ & $\bullet \bullet \bullet$ & $\bullet$ & $\bullet$ & $\bullet$ & $\bullet \bullet$ \\
\hline 8. Scheers 666 & $\bullet \bullet$ & $\bullet$ & $\bullet$ & $\bullet$ & - & $\bullet$ & - \\
\hline 9. LT 8319 & $\bullet \bullet$ & $\bullet \bullet$ & $\bullet$ & $\bullet$ & $\bullet$ & 0 & $\bullet$ \\
\hline
\end{tabular}

Tabl. 11. Les potins dans les fosses de Manching, référence $(K)$ : Kellner 1990, p. 35 sq, d'après la typologie Scheers, 1977 (S) et Geiser, Gruel (GT).

\begin{tabular}{|c|c|c|}
\hline Type & Matériel associé & Référence \\
\hline S $658 \mathrm{ff}$. & trouvailles avant LT D1 & $\mathrm{K} n \circ 11$ \\
\hline S $658 \mathrm{ff}$. & 1/4 céramique peinte, as romain 145/138 avant J.-C. & $\mathrm{K} n \circ 10$ \\
\hline GT A 2 & céramique & $K n \circ 19$ \\
\hline GT A 2 & env. $10 \%$ céramique peinte & $\mathrm{K} \mathrm{n}^{\circ} 17$ \\
\hline GT A $1 / 2$ & env. $40 \%$ céramique peinte, fibule type Nauheim & $\mathrm{Kn} \mathrm{n}^{\circ} 18$ \\
\hline GT A 6,1 & 2 pièces céramique peinte, céramique fine lignée & $\mathrm{Kn} \mathrm{n}^{\circ} 26$ \\
\hline GT A 4,2 & $15 \%$ céramique peinte & $\mathrm{K} \mathrm{n}^{\circ} 24$ \\
\hline GT A 5 & $\begin{array}{l}30 \% \text { céramique peinte, fibule type Nauheim } \\
\text { moules en céramique }\end{array}$ & $\mathrm{K} \mathrm{n}^{\circ} 27$ \\
\hline LT 9361 & céramique peinte, céramique fine peignée & $K n \circ 34$ \\
\hline
\end{tabular}

timoine sont détectés en quantité mineure ou seulement en traces. Le zinc, le bismuth, le manganèse, le cobalt ne sont pas détcctés ou sculcment identifiés sous forme de traces. Bien que les trois types de potins analysés se ressemblent beaucoup dans leur composition, il y a cependant des différences dans le détail. Ainsi, la monnaie $n^{\circ} 2$ contient seulement des traces de plomb. Les éléments arsenic et antimoine qu'on a détectés sont présents en grande quantité dans les $n^{\circ} 1$ et 3 (mêmes types) et manquent dans la monnaie $n^{\circ} 2$. Le $\mathrm{n}^{\circ} 2$ a aussi une apparence différente, car il présente une couleur dorée rougeâtre. La teneur en étain est réduite de moitié (environ $27 \%$ ) par comparaison avec d'autres monnaies analysées (en moyenne $50 \%$ ). Par contre la teneur en étain du potin du 
type LT $8319\left(\mathrm{n}^{\circ} 9\right)$ est supérieure à la moitié (environ $67 \%$ ). La couleur de la pièce est semblable à celle d'une monnaie d'argent. Une monnaie du type Scheers 666 classe $I_{j}\left(n^{\circ} 7\right)$ a une teneur d'antimoine élevée (environ $15 \%$ ), ce qui correspond à six fois la quantité trouvée dans les autres pièces analysées (environ $2 \%$ ).

Pour la datation des potins trouvés pendant les fouilles de Manching, je peux seulement résumer la situation, car on a découvert peu de potins dans un contexte archéologique bien défini (tabl. 11). La masse provient de trouvailles fortuites.

Ia découverte de potins en fouille dans des habitats en Gaule avec un matériel de l'horizon IT D1 et de la période césarienne-augustéenne pose la question de savoir si la circulation monétaire finit à Manching et s'il y a des corrélations avec les résultats des fouilles en France (Brunaux, Gruel, 1987). Aujourd'hui on peut seulement constater, que les monnaies celtiques, qui sont caractéristiques de la fin de La Tène D1 et du temps de César et d'Auguste et qu'on trouve aussi dans les fortifications romaines comme par exemple Augsburg-Oberhausen (Kellner, 1977) manquent à Manching. On n'a trouvé ni Regenbogenschüsselchen en bronze LT 9442, ni bronzes des Aduatuci LT 8865-8881 ou pièces de Germanus Indutilli LT 9248. Cet indice correspond bien aux recherches de R. Gebhard, qui place, grâce aux types de fibules, la fin de l'oppidum de Manching pendant les années 50-30 avant J.-C. (Gebhard, 1991, p. 100-104).

À l'aide de nouvelles découvertes à Manching et dans d'autres habitats latèniens comme Stöffling ou Egglfing et en Gaule, nous espérons bientôt trouver une solution plus simple de la chronologie des potins de Bavière.

Bernward ZIEGAUS

\section{Bibliographie}

GEBHARD R.

1991, Die Fibeln aus dem Oppidum von Manching, Stuttgart (Die Ausgrabungen in Manching, 14).

HAHN W.

1976, Die Fundmünzen der Römischen Zeit in Österreich, Abt. III, Niederösterreich, Vol. I Carmuntum, Wien.

KELLNER H.J.

1977, Die keltischen Münzen von Augsburg-Oberhausen, Jahrbuch für Numismatik und Geldgeschichte, 27, p. 21-27.

KOBLIT7, H.

1918, Beiträge zur keltischen Münzkunde Böhmens, Milleilungen der Österreichischen Gesellschaft für Münz- und Medaillenkunde, 14, $\mathrm{n}^{\circ} 10$, p. 97-100, $\mathrm{n}^{\circ} 12$, p. 115-117.

NEUMANN G., KiLLNER H.J.

1966, Die keltischen Münzfunde in Mitteldeutschland, Ausgrabungen und Funde, 11, p. 253-260.

Píc J.I.

1903, Cechy na úsvite dejin. Starozitnosti zeme Ceské, 2. Hradiste u Stradonic jako historické Marobudum, Praha.

PROKISCH B.

1993, Keltische Fundmünzen aus Oberōsterreich. Die römischen Münzen des Stadtmuseums Nordico in Linz, Linzer Archäologische Forschungen, Sonderband X, Linz.

\section{RADOMERSKY P.}

1955, Nalezy keltskych mincí v Cechách na Morave a ve Slezsku, in : E. Nohejlova-Pratova, Nalezy keltskych minci v Cechách na Morave a ve Slezsku I 1, Praha, p. 35-69.

ZIEGAUS B.

1989, Der latènezeitliche Münzumlauf in Franken, Bayerische Vorgeschichtsblätter, 54, p. 69-135. 